\title{
Relation Extraction from Biomedical and Clinical Text: Unified Multitask Learning Framework
}

\author{
Shweta Yadav, Srivatsa Ramesh, Sriparna Saha, and Asif Ekbal
}

\begin{abstract}
Motivation: To minimize the accelerating amount of time invested on the biomedical literature search, numerous approaches for automated knowledge extraction have been proposed. Relation extraction is one such task where semantic relations between the entities are identified from the free text. In the biomedical domain, extraction of regulatory pathways, metabolic processes, adverse drug reaction or disease models necessitates knowledge from the individual relations, for example, physical or regulatory interactions between genes, proteins, drugs, chemical, disease or phenotype.

Results: In this paper, we study the relation extraction task from three major biomedical and clinical tasks, namely drug-drug interaction, protein-protein interaction, and medical concept relation extraction. Towards this, we model the relation extraction problem in a multi-task learning (MTL) framework, and introduce for the first time the concept of structured self-attentive network complemented with the adversarial learning approach for the prediction of relationships from the biomedical and clinical text. The fundamental notion of MTL is to simultaneously learn multiple problems together by utilizing the concepts of the shared representation. Additionally, we also generate the highly efficient single task model which exploits the shortest dependency path embedding learned over the attentive gated recurrent unit to compare our proposed MTL models. The framework we propose significantly improves over all the baselines (deep learning techniques) and single-task models for predicting the relationships, without compromising on the performance of all the tasks.
\end{abstract}

Index Terms_-Protein Protein Interaction, Drug Drug Interaction, Medical Concept Relation, Adversarial Learning, Deep Learning, Natural Language Processing, Relation Extraction.

\section{INTRODUCTION}

Owing to the rapid growth of the scientific literature, majority of the available biological facts remain concealed in the form of scientific literature. Over the last two decades, MEDLINE size has risen at a compounded annual growth pace of 4.2 percent. MEDLINE currently holds more than 26,000, 000 records from 5639 publications, which is more than 5 millions than those indexed in 2014 alone. This similar trend was also observed in case of the healthcare data. IBM reported that nearly 2.5 quintillion bytes of the healthcare data are generated globally. Encapsulated within this unstructured text is an enormous amount of significant healthcare and the biomedical data, which are valuable sources of information for the Biomedical Natural Language Processing (BioNLP) domain.

As a consequence of the exponential rise [1], [2] and complexity of the biological and clinical information, it is imperative to advance the automatic extraction techniques to assist biologist in detecting, curating and maintaining databases and providing automated decision support systems for the health professional. This has led to a rise in the interest of the BioNLP community to automatically detect and extract information from the scientific literature and clinical records [3], [4], [5].

Relation extraction is one such task that aims to detect and characterize the semantic relationship between the biologi$\mathrm{cal} / \mathrm{clinical}$ entities. The relation types could vary depending upon the genres and domains, such as interactions between genes, proteins, drugs, or medical concepts (problems, treatments, or tests).

In this paper, we study the relation extraction (RE) on the most popular biomedical and clinical tasks, namely drug-drug

- All the authors are with the Department of Computer Science and Engineering, Indian Institute of Technology Patna, Bihar, India, 801103.

E-mail: (shweta.pcs14,sriparna,asif)@iitp.ac.in interaction (DDI), protein-protein interaction (PPI) and clinical relation. DDI detection is an significant area of patient safety studies as these interactions can become very hazardous and boost the cost of health care. Similarly, the knowledge about interaction among proteins can help in understanding biological processes, complex diseases, and guiding drug discoveries [6]. In the clinical domain, the ability to recognize relations among medical concepts (treatments, tests, or problems) allows the automatic processing of clinical texts, resulting in clinical decision-making, clinical trial screening, and pharmacovigilance.

In the vast literature on relation extraction, several techniques have been proposed to solve the problem ranging from the semanticinjected kernel model [7], [8] to the machine learning embedded models [9], [10]. In recent past, with the success of deep neural networks, the state-of-the-art models for these tasks have been drifted towards the deep learning frameworks [11], [12].

However, there have been a very few attempts in improving the performance of RE system irrespective of the tasks or domains. One potential solution is to model the relation extraction problem in the multi-task learning framework, where a problem together with the other related problem can be learned by leveraging the shared representation. This method of multi-task learning provides advantages in (1) minimizing the number of parameters and (2) reducing the risk of over-fitting. The aim of multi-task learning (MTL) is to enhance the system performance by integrating the other similar tasks [13], [14]. When tasks are common and, in particular, when training data is limited, MTL can contribute to better results than a model trained on a single dataset, allowing the learner to capitalize on the commonality among the tasks. This can assist the overall model as dataset can contain information which are complementary to address individual tasks more correctly when trained jointly [15].

However, most of the existing methods on multi-task classi- 
fication tend to divide the feature space of each task into shared and private, depending solely on hard-parameter or soft-parameter sharing. As such, these task-shared features are prone to being contaminated with external noise and task-specific features that often lead the system to suffer from the redundancy of the feature.

To combat the contamination issue of task-shared features, in this paper, we propose an adversarial multi-task relation extraction framework. This framework deals with utilizing concept of adversarial learning to inherently disjoint the task-specific and taskinvariant feature spaces. In adversarial learning [16], a model is trained to correctly classify both unmodified data and adversarial data through the regularization method. The adversarial learning paradigm provides an assurance that task-shared feature space is not contaminated with task-specific features and contains only task-invariant features.

In our study, we use the bi-directional gated recurrent units (Bi-GRU) [17] as a learning algorithm which has the capability to learn the features by capturing the long dependency information. Bi-GRU, unlike other Recurrent Neural Networks (RNN), such as Long Short Term Memory (LSTM) is computationally less expensive [18]. GRU also has the gating mechanism similar to LSTM to control the information flow. But unlike LSTM, GRU has no memory unit and has an update and reset gate.

As such, when down-sampling operation is performed on the output of GRU, we extract the optimal features from the entire input sequence covering the complete context information. In the literature, the attention mechanism has shown the promising results in relation extraction by generating the optimal and effective features. In attention mechanism, a simple strategy has been followed by computing a weight vector corresponding to each hidden state of the RNN or CNN. The final hidden states are computed by performing the pooling operation ( $\max , \mathrm{min}$, average etc.) on the weighted representations of hidden states. However, the computed attention weight focuses on a specific aspect of the input sequence. In this work, we attempt to capture multiple aspects of the input sequence by exploiting the self-attention mechanism. Basically, we learn to generate the multiple attention weight vectors, which eventually generate the multiple final representations of hidden states considering the various aspects of the input sequence.

We apply our proposed approach on four popular benchmark datasets namely, AiMED \& BioInfer for PPI [19], DrugDDI for DDI [20], and $2010 \mathrm{i} 2 \mathrm{~b} 2 / \mathrm{VA}$ NLP challenge dataset for clinical relation extraction (MCR) [21]. Our proposed MTL model obtained the F1-Score points of 77.33, 76.33, 72.57, and 81.65 for AiMed, BioInfer, DDI and i2b2 relation extraction dataset, respectively. We observe an average 5\% improvement in F-score in comparison to single task learning baseline model and over 3\% improvement on MTL baseline model. Performance on any dataset does not decrease considerably, and performance increases significantly for all the four datasets. These are promising outcomes that set the potential for using the MTL model to solve the issue of biomedical RE. In addition to the baselines, our proposed model outperforms the state-of-the-art methods for all the datasets. This shows that, when we have tasks in common, multi-task can assist over the single-task models. The contributions of our proposed work can be summarized as follows:

1) We propose a multi-task learning (MTL) framework for relation extraction that exploits the capabilities of adversarial learning to learn the shared complementary features across the multiple biomedical and clinical datasets. We also exploit the self-attention mechanism which allows the final feature representation to directly access previous Bi-GRU hidden states via the attention summation. Therefore, the Bi-GRU does not need to carry the information from each time step towards its last hidden state.

2) Our proposed model is capable of automatically extracting the various relations (such as Protein-Protein Interaction, Drug- Drug interaction: 'int', 'advice', mechanism, effect, and relation between medical problem and treatment, test and treatment, treatment and treatment).

3) We validate our proposed framework on four popular benchmark datasets (AiMED, BioInfer, SemEval 2013 Drug Interaction task, and i2b2 medical relation shared task dataset) for relation extraction, having different annotation schemes.

4) Our unified multi-task model achieves the state-of-the-art performance and outperforms the strong baseline models for all the tasks in the respective datasets.

\section{Related Works}

There has been recent surge in the interest of the BioNLP community to automatically detect and extract information from the scientific literature and clinical records [22], [23], [24], [25], [26], [27. In the past decade, there has been tremendous amount of the work on varieties of the relation extraction task such as extracting relationship between the bio-entities (proteins, gene, diseases, etc.) from the biomedical literature. Much previous works are done by using the Kernel-based technique which allows the representation learning of the data in the form of dependency structures and syntactic parse trees. Some of the other prominent techniques for extracting the relationships are based on the pattern-matching technique. Recently, with the success of deep learning technique, several techniques based on the Convolutional Neural Network, Recurrent Neural Network, and Long Short Term Memory network are widely utilized for extracting the relationships from biomedical literature and clinical records. Based on the tasks, we divide the related works in the following three categories:

- Protein Protein Interaction task: Several NLP techniques have been proposed to identify the relationships between the protein entities. The preliminary studies [28], [29], [30] on this task were essentially solved by using pattern-based model, where patterns were extracted from the data based on their syntactic and lexical properties. The main drawback with this approach is the inability to properly handle the complex relationship expressed in coordinating and relational clause. Dependency based approaches [31], [32] are more syntax aware techniques and have broader coverage than naive pattern based approaches. Some of the studies [33 exploring the dependency based techniques incorporate the dependency information as a shortest dependency path between the sentences. Also the technique based on kernel method is often explored in the area of PPI extraction. Some of the prominent kernel-based approaches for extracting the PPI include edit-distance kernel [33], bag-of-word kernel [34], all-path kernel [8], graph kernel [35], and tree-kernel [36]. [37] proposed a walk-weighted sub sequence kernel that captures the syntactic structure by matching the e-walk and v-walk on the shortest dependency path. [38] proposed a technique based on convolutional tree kernel by integrating the patterns of protein interaction.

Recently, various studies have exploited deep learning based 
techniques [39], [40] which do not require the manual feature engineering unlike the previous techniques based on the kernel, pattern and dependency based methods. [41] first proposed the deep learning technique for extracting the relationship between the protein pairs. They used the $\mathrm{CNN}$ as a base learner over the word embedding generated through the Google News corpus. [42] proposed a neural network framework which integrates several lexical, semantic and syntactic level information in the CNN model. Their study shows that integrating these additional information provides very minor improvement overall. [43] in their study proposed a two channel CNN technique for high level of feature extraction. In the first channel, they used words with additional syntactic features like part-of-speech, chunking information, dependency information, named entities and the word position information w.r.t the protein entities. In the second channel, they used the parent word information for every word. [12] propose a greedy layer-wise unsupervised technique to extract the PPIs. They utilized the auto encoder on the unlabelled data for the parameter initialization of the deep neural network model and applied the gradient decent method using back propagation to train the whole network. Various studies [40] on PPI extraction task have also explored the Recurrent Neural Network framework. [44] proposed a method based on the Bi-directional Long Short Term Memory Network (Bi-LSTM) equipped with the stacked attention mechanism. The input to their model is the shortest dependency path between the entity pairs. Their study shows that providing multiple attentions can assist the model in better capturing the long contextual and structural information. [45] proposed a tree RNN with structured attention framework for extracting the PPI information.

- Drug Drug Interaction task: Existing techniques on drug drug interaction can be categorized into one-stage and twostage classification scheme [46]. In the one-stage classification [47], [48], the aim of the task is to identify the multiple relationships between the drug pairs, which could be from any of the interacting class or negative class. Several methods have explored the multi-class classifier, to capture the relationship between two target drugs in a sentence. While, in the two-stage classification [49], [50] scheme, there are two steps. The first step determines whether the target drug pair is interacting/non-interacting. In the second step, only the interacting sentences are considered as inputs to the multiclass classifier. These approaches can further be classified into handcrafted feature and latent feature based methods. Techniques [51], [52], [53] based on the hand-crafted feature mainly utilize support vector machines (SVMs) as the base learner. These techniques are reliant on several handcrafted features such as Part-of-Speech, chunk, syntax trees, dependency parsing, and trigger words. These methods are utilized in other biomedical relation extraction tasks, such as adverse drug reactions extraction tasks [54], [55], protein protein interaction extraction [56], relation extraction between diseases and genes [57], and relations between the medical concepts [58]. These techniques have appeared to perform well, however they are very domain-specific and are dependent on other NLP tools. Approaches exploring the latent features, are based on deep learning models, that are proved to be powerful solutions to the feature based models. Below we provide the survey in detail for the above described methods:

- Linear Methods: This method utilizes a linear classifier that takes as an input the domain specific or manually designed features. The system proposed by Uturku [49] explored Turku event extraction system (TEES) for identifying the drug interaction pairs. TEES utilizes the features of dependency parsing and lexicon derived from MetaMap and Drugbank. |50| developed two-stage classification technique based on SVM. They explored several handcrafted features such as lexical, contextual, semantics and tree structured features.

- Kernel Methods: These techniques are more advanced than linear methods, where they have explored graph based features. All-paths graph kernel [59] learns a classifier based on the weighting scheme for dependency parse tree feature and surface feature. k-band shortest path spectrum [60] utilizes the shortest dependency path between the entity pairs to build a classifier. It further permits the mismatches for variables and includes all the nodes within $\mathrm{k}$-distance from the shortest path. The shallow linguistic kernel [61], as the name suggests, captures the shallow linguistic features such as part-of-speech, stem, word and other morphological features that also explore the properties of the surrounding words. [62 proposed an ensemble based DDI extraction system. They explored the various kernel classifiers in addition to the case-based reasoning technique for classifying the drug pairs. [53] also explored various kernel classifier by integrating multiple kernel methods such as SL kernel, mildly extended dependency tree (MEDT) kernel and path-enclosed tree (PET) kernel. PET kernel captures the smallest subtree involving the two entities in a phrase structure parse tree. MEDT kernel uses linguistically motivated expansions to capture the prominent clue words between the entity pairs. The system proposed by [63] also utilizes several kernels based on the feature and tree kernel methods. Precisely, they explored MEDT, SL, PET, global context and local context kernel.

- Deep Learning Technique: Deep learning technique is based on the neural network approach that utilizes latent features instead of hand-crafted features. This technique encodes the word level representation using neural network for the generation of sentence level features. For the final classification, the network uses the sentence level feature. In the recent past, several deep learning methods have been used to address DDI tasks. SCNN system was proposed by [64]. They have used convolution neural network to capture more dense sentence representation feature. SCNN also utilized some additional features such as PoS and dependency tree based feature. [46] advanced the stateof-the-art technique by proposing three different models based on the concept of LSTM and attentive pooling. All of these models takes as an input the word and position embedding and does not rely on any hand-crafted features. Some of the other prominent work that has explored deep learning framework are [65], [66], [67], [68], |69]

- Medical Concept Relation Extraction task: Electronic medical records such as patient's discharge summaries and progress notes contains information about the medical concepts and relationship. To aid an advanced patient care, it is required have a technique for automatic processing of 
the clinical records. To address this issue, Informatics for Integrating Biology \& the Bedside (i2b2) organized a shared task challenge [21] that aims to identify the relationship between medical concepts i.e. problems, treatments, or tests from the EMR document. Identifying the correct relationship between the medical concepts requires the knowledge of the context in which two concepts are discussed. Existing techniques for extracting the relations between the medical concepts can be grouped into semi-supervised and supervised classes. [70] explored the semi-supervised method to determine the relationship between a concepts-pairs. They used maximum entropy as an classification algorithm to train separately three classifier for each concept pair i.e. test-problem, treatment-problem, and problem-problem relations. They explored various external features obtained from other NLP pipeline such as cTAKES and MetaMap. Additionally, they also used word-level feature, PoS tags, dependency path based feature, and distance feature that capture minimal, average and maximal tree distances to the common ancestor. To overcome the label imbalance problem in the training data, they computed the relatedness between two medical concepts by using pointwise mutual information in MEDLINE and bootstrapping with unlabeled examples.

Majority of the research work has been carried out on this problem are highly dependent on the supervised approach. In those works [71], [72], [73], statistical machine learning classifier (CRF, SVM) is used to identify the relations. As the i2b2 shared task dataset, contained a large portion of concept pairs without any relations, some of the system [74], [75], [76] proposed two stage classification methods, where in the first stage, the concept pair with relation/no relation were classified. In the latter stage, only those concept pair with the relation participate for identifying the given relationship. All of the participating system heavily rely on the hand-crafted features. They exploited semantic, lexical, syntactic, domainspecific ontology feature. The system proposed by [77] used medical knowledge graph (UMLS) concept identifiers and applied feature selection technique to capture more relevant feature. [73] used linguistic feature to complement their machine learning component to extract the medical concept relations. Recently, neural network techniques are widely adopted for the clinical relation extraction task. |78| explored the capabilities of convolutional neural network to capture prominent feature for extracting relations. Another study conducted by [79] used two variant of RNN-LSTMs network, segment level LSTM and sentence level LSTM for encoding the relation. The experimental results shows that the proposed approach perform comparable w.r.t state-of-the-art system. They also identified that word embedding from clinical text is more beneficial than the general text.

Other prominent study was conducted by [80] to identify the relations among disease and treatment. They used several neural network and graphical models. Furthermore, they utilized other hand-crafted features such as lexical, semantic, and syntactic feature for classification. They conducted their study on very small dataset consisting of biomedical research article. [81] applied CRF for extracting relations between disease, treatment and gene. They proposed two step model where in the first step, they identified the entities and in the second step, they extract the relationship. For both the steps, they explored CRF as the base learner. [57] developed a system for finding the association between disease, drug and target in EU-ADR dataset. They exploited kernel based method that uses the shallow linguistic and dependency kernel for extracting the relations. [82] developed shortest dependency path based deep neural network framework that also utilizes other features like Parts-of-speech information, dependency labels, and the types of the entities.

\section{MATERIALS AND METHODS}

In this section, we describe the methodology used to extract the relations from various biomedical texts in our proposed multi-task learning framework. We begin by the problem statement followed by the introduction of the Gated Recurrent Unit (GRU) which is used in our models as a base learning algorithm. Following that, we describe the proposed multi-task model in detail that utilizes the concept of adversarial learning.

\section{Problem Statement:}

Given an input text sequence $S$ consisting of $n$ words, i.e., $S=$ $\left\{w_{1}, w_{2} \ldots w_{n}\right\}$ and a pair of entities $1\left(e_{1}, e_{2}\right)$ where $e_{1} \in S$ and $e_{2} \in S$. The task is to predict the maximum probable class $\bar{y}$ from the set of class labels, $Y$. Mathematically,

$$
\bar{y}=\underset{y \in Y}{\arg \max } \operatorname{prob}\left(y \mid S, e_{1}, e_{2}, \theta\right)
$$

where, $\theta$ is the model parameter. Each token $w_{i} \in S$ of the input sequence $S=\left\{w_{1}, w_{2}, \ldots, w_{n}\right\}$ is mapped into $d$ dimensional word embedding sequence $x=\left\{x_{1}, x_{2}, \ldots, x_{n}\right\}$, where $x_{i} \in$ $\mathcal{R}^{d}$.

\subsection{Gated Recurrent Units}

In this work, we use GRU [17] as the base learner. The GRU is an improved version of the recurrent neural network and being used as variant of the LSTM due to its simpler architecture over the LSTM. Similar to the other RNN, GRU has the capability of internal memory. This internal memory helps them to exhibit temporal dynamic behaviour for a time sequence. Additionally, GRU is able to solve the problem of the vanishing gradient problem which comes with a standard recurrent neural network. Similar to LSTM units, GRU also have the gating mechanism to control the flow of information and produce the effective hidden state representation.

GRU has two neural gates, update and reset gate. The task of update gate is to helps the model for determining the amount of information need to be carry forward along to the future. The reset gate helps the model to determine the amount of past information need to be forget. Specifically, a GRU network successively reads the input token $x_{i}$, as well as the visible state $h_{i-1}$, and generates the new states $c_{i}$ and $h_{i}$.

$$
\begin{aligned}
z_{i} & =\sigma\left(\mathbf{W}^{z} x_{i}+\mathbf{V}^{z} h_{i-1}+\mathbf{b}^{z}\right) \\
r_{i} & =\sigma\left(\mathbf{W}^{r} x_{i}+\mathbf{V}^{r} h_{i-1}+\mathbf{b}^{r}\right) \\
c_{i} & =\tanh \left(\mathbf{W} x_{i}+\mathbf{V}\left(r_{i} \odot h_{i-1}\right)+\mathbf{b}\right) \\
h_{i} & =z_{i} \odot c_{i}+\left(1-z_{i}\right) \odot h_{i-1}
\end{aligned}
$$

where $z$ and $r$ are the update and reset gates, respectively. The final representation at a given time $t$, from a bi-directional GRU

1. The entity pair can be the protein, disease, problem name, etc. 


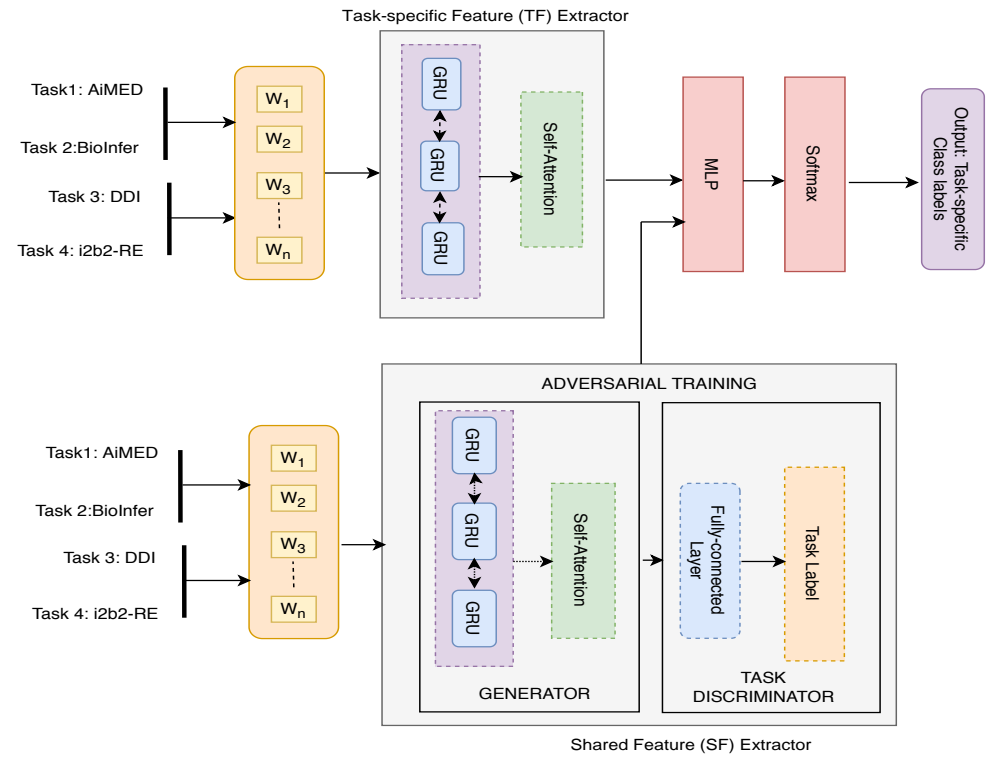

Fig. 1: Proposed multi-task model for various biomedical relation extraction tasks.

(Bi-GRU), can be computed by concatenating the forward $\overrightarrow{h_{t}}$ and backward $\overleftarrow{h_{t}}$ hidden states. From here onward, we will call Bi-GRU as function having the inputs, $x_{t}$ and $h_{t-1}$ and output $h_{t}$.

\subsection{Relation Extraction Framework}

In the relation extraction framework (c.f. Figure-1) the input sequence $x=\left\{x_{1}, x_{2}, \ldots, x_{n}\right\}$ with the corresponding entity pair $\left(e_{1}, e_{2}\right)$ is transformed into the hidden state representation using the Bi-GRU (c.f. section 3.1. In order to emphasize the given entity pair, the corresponding entity word in the input sequence is marked with the special token ENTITY and assigned a fixed word embedding to it. More formally, the hidden state at each time step is calculated as follows:

$$
h_{t}=\operatorname{Bi}-\operatorname{GRU}\left(x_{t}, h_{t-1}\right)
$$

Let the hidden state dimension for each Bi-GRU unit be $d_{h}$. We formulate a hidden state matrix $H \in \mathbb{R}^{n \times d_{h}}$.

$$
H=\left(\mathbf{h}_{\mathbf{1}}, \mathbf{h}_{\mathbf{2}}, \cdots \mathbf{h}_{\mathbf{n}}\right)
$$

We compute the effective input sequence encoding $\bar{h}$ using function ${ }^{2}$ fun $(\bar{h} ; \theta)$ with learning parameter $\theta$. The input sequence encoding $\bar{h}$ is fed to a fully connected softmax layer to generate the probability distribution over the predefined classes.

$$
\bar{y}=\operatorname{softmax}\left(\bar{h}^{T} \mathbf{W}+\mathbf{z}\right)
$$

Here, $\mathbf{W}$ and $\mathbf{z}$ are weight matrix and bias vector, respectively. The term $\bar{y}$ denotes the predicted probability distribution.

Let us have a training dataset with $N$ samples such that $\left\{\left(x_{1}, y_{1}\right),\left(x_{2}, y_{2}\right), \ldots,\left(x_{n}, y_{n}\right)\right\}$. The network parameters are trained to minimize the loss function- cross entropy of the probability distributions of predicted $(\bar{y})$ and true class $(y)$ over the $C$ number of classes.

$$
\mathcal{L}_{\mathcal{C E}}(\bar{y}, y)=-\sum_{i=1}^{n} \sum_{j=1}^{C} y_{i}^{j} \log \left(\bar{y}_{i}^{j}\right)
$$

2. This function is adversarial learning based self-attentive network which computes the effective input sequence encoding.

\subsubsection{Attentive Mechanism:}

We encode the input sequence with by adopting the self-attentive mechanism [83] over the Bi-GRU generated hidden state sequence. The input to the attention mechanism is the Bi-GRU hidden states $H$. The self-attention generate the attention weight vector $v$ computed as follows:

$$
\begin{aligned}
& \mathbf{p}=\tanh \left(U H^{T}\right) \\
& \mathbf{v}=\operatorname{softmax}(\mathbf{w} p)
\end{aligned}
$$

Here $U \in \mathbb{R}^{d_{a} \times d_{h}}$, and $\mathbf{w} \in \mathbb{R}^{d_{a}}$ and $d_{a}$ is a hyperparameter and $d_{h}$ is the size of hidden state. The final hidden state representation $s$ is computed by the weighted (weight provided by $\mathbf{v}$ ) sum of the each time step of the Bi-GRU. The major drawbacks of the aforementioned representation is that it focuses on the specific component of the input sequence. The specific component could be a relation between a given entity to other words in the sequence. We call it is a aspect to represent the input sentence. In order to capture the multiple aspects, we required multiple m's that capture the various notion of the input sequence. Therefore, we extend the attention mechanism from focusing on single aspect to multiple aspects. Let us assume, we want to extract ' $a$ ' number of multiple aspects from the input sequence. To achieve this, we extend the $\mathrm{Eq} 7$ as follows:

$$
\begin{aligned}
\mathbf{p} & =\tanh \left(U H^{T}\right) \\
\mathbf{V} & =\operatorname{softmax}(\mathbf{W} p)
\end{aligned}
$$

Here, $\mathbf{W} \in \mathbb{R}^{a \times d_{a}}$. Formally, We compute the ' $a$ ' weighted sums by multiplying the matrix $V$ and LSTM hidden states $H$. We obtain a matrix representation $M$ of the input sequence by multiplying the attention weight matrix $V$ and hidden state representation $H$.

$$
M=V H
$$

We concatenate each row of the matrix representation to get the final vector representation of the input sequence. 


\subsection{Multi-task Learning for Relation Extraction}

In this study, we introduce the novel method for biomedical relation extraction exploiting the adversarial learning in the multitask deep learning framework. Our model leverages joint modeling of the entities and relations in a single model by exploiting attentive Bi-GRU based recurrent architecture. We propose an adversarial multi-task learning with attention (Ad-MTL) model for relation extraction task. Multi-task learning exploits the correlation present among similar tasks to improve classification by learning the common features of multiple tasks simultaneously. We build a latent feature space that holds the features that are common to various tasks. Specifically in the model, the outputs generated at each time-step of the shared Bi-GRU, are considered to be common latent features. We generate the task-specific feature features for each task by task-specific Bi-GRU network equipped with the self-attentive network discussed in section 3.2.1

We compute the two hidden states at each time step $t$ for a given task $k$, one task-specific hidden state, $h_{t}^{k}$, and another shared hidden state, $s_{t}^{k}$. The former captures the task dependent features and the latter captures the task invariant features. Both the hidden state representations are computed similar to Eqn. 3 .

$$
\begin{aligned}
s_{t}^{k} & =\operatorname{BI-GRU}\left(x_{t}^{k}, s_{t-1}^{k}, \theta_{s}\right) \\
h_{t}^{k} & =\operatorname{BI-GRU}\left(x_{t}^{k}, h_{t-1}^{k}, \theta_{h}\right)
\end{aligned}
$$

where $\theta_{s}$ and $\theta_{h}$ are Bi-GRU's parameters, $x_{t}^{k}$ denotes the input at time $t$. We generate the task-specific feature representation by applying self-attention using equation 9 . We also use a feedforward network with a hidden layer to project the attentive feature representation to another vector space. We call the final taskspecific feature representation as of task $k$ as $T F_{k}$. Similar to the task-specific feature generation, we use a feed-forward network to project the the shared feature into another vector space and call it is a shared feature $S F$. The concatenation of the shared and task specific features is fed into a fully connected layer followed by the softmax layer. The softmax layer returns the class distributions, $y_{\text {pred }}^{k}$, for the underlying task, $k$. For every task, $k$, with training samples $\left(x_{i}^{k}, y_{i}^{k}\right)$, both the task specific parameters and shared parameters are optimized to minimize the cross-entropy of the predicted $\left(\hat{y}_{i}^{k}\right)$ and actual probability distributions $\left(y_{i}^{k}\right)$, whose loss is computed as:

$$
\mathcal{L}_{C E}^{k}=\mathcal{L}_{C E}\left(\hat{y}_{i}^{k}, y_{i}^{k}\right)
$$

where $\mathcal{L}_{C E}(\hat{y}, y)$ is defined as Eq. 6 .

\subsubsection{Adversarial Training}

The above discussed model though intended to separately host the shared and task-specific features, provides no guarantee to behave so, there might be contamination of shared features in the taskspecific feature space and vice versa. To handle this, we exploited the principle that a good shared feature space has features that make it impossible to predict the source task of the feature. For achieving the above, a Task Discriminator $D$ is used to map the attention prioritized shared feature to estimate the task of its origin. In our case, Task Discriminator is a fully connected layer using a softmax layer to produce the probability distribution of the shared features belonging to any task. A BiGRU works as Generator $(G)$ to generate shared features. This Bi-GRU layer is made to work in an adversarial way, preventing the discriminator from predicting the task and hence preventing contamination in the shared space. The adversarial loss is used to train the model. Similar to [16], [84], we use the following adversarial loss function

$$
\mathcal{L}_{a d v}=\min _{G}\left(\max _{D}\left(\sum_{t=1}^{T} \sum_{i=1}^{N_{t}} d_{i}^{t} \log \left[D\left(G\left(x^{t}\right)\right)\right]\right)\right)
$$

where $d_{i}^{t}$ is the gold label indicating the type of the current task. The min-max optimization problem is addressed by the gradient reversal layer [85]. The total loss of the network will be as follows:

$$
\mathcal{L}_{\text {total }}=\alpha \sum_{k=1}^{K} \mathcal{L}_{C E}^{k}+\beta \mathcal{L}_{a d v}
$$

where $\alpha$ and $\beta$ are the scalar parameters.

\section{Experimental Results and Discussion}

In this section, we will begin by briefly describing the various tasks and the datasets followed by the experimental results and analysis. In this study, we focus on the following tasks:

\subsection{Protein Protein Interaction Extraction (PPI):}

The goal of this task is to classify whether or not a sentence containing a proteins pair actually indicates interaction between the pair. Here, we considered the positive instances as the interacted protein-pair and the negative instances as the non-interacted protein pair. If the relationship between the protein pair is not explicitly provided, the pairs were considered to be the negative instances. In order to identify those instances, we extracted all possible proteins pairs from the sentences.

We utilize two standard benchmark datasets for PPI tasks, namely AiMed and BioInfer AiMed dataset is derived from the $197 \mathrm{ab}-$ stracts of the Database of Interacting Proteins (DIP) and contains manually tagged relationship between the protein entities. There are in total 5834 relationship out of which 1000 are interacting relation and 4834 non-interacting relation.

BioInfer (Bio Information Extraction Resource) is another manually annotated PPI dataset developed by Turku BioNLP grouf 4 that contains over 1100 sentences. It has 2534 instances of protein interacting relationship and 7132 non-interacting relationship.

\subsection{Clinical Relation Extraction (MRE):}

This task aims to extract the relation between the clinical entities (Problem, Treatment, and Test) from the EMR. For this, we used the benchmark dataset released by i2b2 as a part of i2b2 2010 clinical information challenge [21]. The dataset was collected from three different hospitals, which consists of discharge-summaries and progress notes of the patients those were manually annotated by the medical practitioners for identifying the three major relation types: medical problem-treatment $(\mathrm{TrP})$ relations, medical problem-test (TeP) relations, and medical problem-medical problem (PP) relations. These relations were further fine-grained into 8 different relation types which were: treatment caused medical problems $(\operatorname{TrCP})$, treatment administered medical problem (TrAP), treatment worsen medical problem (TrWP), treatment improve or cure medical problem (TrIP), treatment was not administered because of medical problem (TrNAP), test reveal medical problem (TeRP), Test conducted to investigate medical problem (TeCP), and Medical problem indicates medical problems (PIP). The exact

\footnotetext{
3. http://corpora.informatik.hu-berlin.de/

4. http://bionlp.utu.fi/
} 
definition of each of these relation types can be found in [21]. 5 It is to be noted that since we did not have enough training samples for all relation classes present in the dataset, we have removed following three classes: TrWP, TrIP, and TrNAP. this same strategy was also followed by the [78].

\subsection{Drug Drug Interaction Extraction (DDI):}

Given a sentence with two pharmacological substances, this task aims to classify if the given drug pair interacts with each other or not. For this task, we utilize the standard benchmark DDI corpus from Semeval 2013 DDIExtraction challenge dataset [20]. The DDIExtraction 2013 task exploits the DDI corpus, which contains MedLine abstracts on drug-drug interactions as well as documents describing drug-drug interactions from the DrugBank database. The corpus consists of total 1,017 abstracts from Medline (233) and DrugBank (784) databases which were manually annotated to obtain 18,491 pharmacological substances and 5,021 drug-drug interactions. Here each interacted drug pair is further classified into one of four types, namely mechanism, advice, effect, and int. It is to be noted that during the challenge, original dataset has 23756 false samples for training and 4737 for testing. But when we obtained the data set from shared task organizers, there were only 22474 false training instances and 4461 testing sample for false class. In the Table-3 we have provided the statistics of the DDI dataset.

The detailed statistics of all the three datasets are reported in the Table-1/2]3

\begin{tabular}{ccc}
\hline Dataset & Interacting Pairs & Non-interacting pairs \\
\hline & & \\
AiMed PPI & 1000 & 4834 \\
BinInfer PPI & 2534 & 7132 \\
\hline
\end{tabular}

TABLE 1: Statistics: AiMed and BioInfer Dataset

\begin{tabular}{ccc}
\hline Label & Relation & No.of Samples \\
\hline 0 & TrIP & 203 \\
1 & TeRP & 3053 \\
2 & TrAP & 2617 \\
3 & PIP & 2203 \\
4 & TeCP & 504 \\
5 & TrCP & 526 \\
6 & TrNAP & 174 \\
7 & TrWP & 133 \\
8 & NONE & 54530 \\
\hline
\end{tabular}

TABLE 2: Statistics: 2010 i2b2/VA NLP Challenge dataset

\begin{tabular}{cccc}
\hline Label & Relation & No.of Samples (Train) & No.of Samples (Test) \\
\hline 0 & False & 22474 & 4461 \\
1 & effect & 1685 & 360 \\
2 & mechanism & 1316 & 302 \\
3 & advice & 826 & 221 \\
4 & int & 188 & 96 \\
\hline
\end{tabular}

TABLE 3: Statistics: Semeval 2013 DDIExtraction challenge dataset

5. While, the actual dataset released during the challenge was having 394 documents for training and 477 documents for testing. However, we were able to download only 170 documents for training and 256 documents for testing as also pointed out by [78] from i2b2 website.

\section{Network Training and Hyper-Parameters SETTING}

We train the network by minimizing the total loss of the network (Eq. 14). In adversarial training, first we pre-trained the discriminator to avoid the instability in the network. To pretrain the discriminator, we use a Bi-GRU network to get the representation of the sentences from the different tasks. We have shown the training process in Algorithm 1] The shared feature extractor model $S$ in Algorithm 1 is a Bi-GRU network followed by self-attention layer and it is being exploited by all the tasks (c.f. Section 3.3. In the shared feature extractor, there is an additional adversarial learning component, where feature extractor (Generator) operates adversarially towards a learnable multi-layer perceptron (Discriminator), preventing it from making an accurate prediction about the types of the task the features generated from. For generating the word embedding, we have used pre-trained embedding available at 6

\subsection{Evaluation Criteria}

We evaluate the performance of all our models using macroaveraged precision, macro-averaged recall and macro-averaged F1-Score metrics. Due to the unavailability of separate validation set for AiMED, BioInfer, and i2b2-2010 clinical relation task datasets, we adopt 10-fold document cross-validation strategy to compute the precision, recall, and F1-score values. We consider the predicted class-label as the correct only if it has the exact match with the ground truth annotation. For the DDI task, we report the performance of the models on the test set.

\subsection{Results and Analysis}

Inspired by the recent success of the deep learning based frameworks in solving the relation extraction task, we develop three strong baselines based on STL and MTL frameworks for the purpose of comparison. Figure-2 provides the architecture for the below described baseline model.

- Baseline 1: The first baseline is a STL model constructed by training a Bi-GRU on the features obtained from the embedding layer to capture the long-term dependencies as defined in Subsection-3 In our experiment, we build the individual model for each dataset.

- Baseline 2: This single-task learning (STL) model is an advanced version of Baseline 1, where the sentence encoder of this model is also equipped with the word-level attention [86].

- Baseline 3: It is a multi-task model with the shared Bi-GRU followed by word-level attention that acts as a shared feature extractor for all the tasks.

The results obtained by our proposed model and the baseline systems for each tasks is reported in Table-4 The results obtained demonstrate the efficiency of our proposed MTL framework over other models that explore state-of-the-art techniques based on a single task and multi-task neural network. For AiMed dataset, our proposed MTL model outperformed the Baseline 1 and Baseline 2 model by 6.96 and 8.64 F1-Score points, respectively. Similar trend was also followed for the BioInfer dataset where, we observe the performance improvements of 5.21 and 7.58 F1Score points over Baseline 1 and Baseline 2, respectively, by the

6. http://evexdb.org/pmresources/vec-space-models/ 

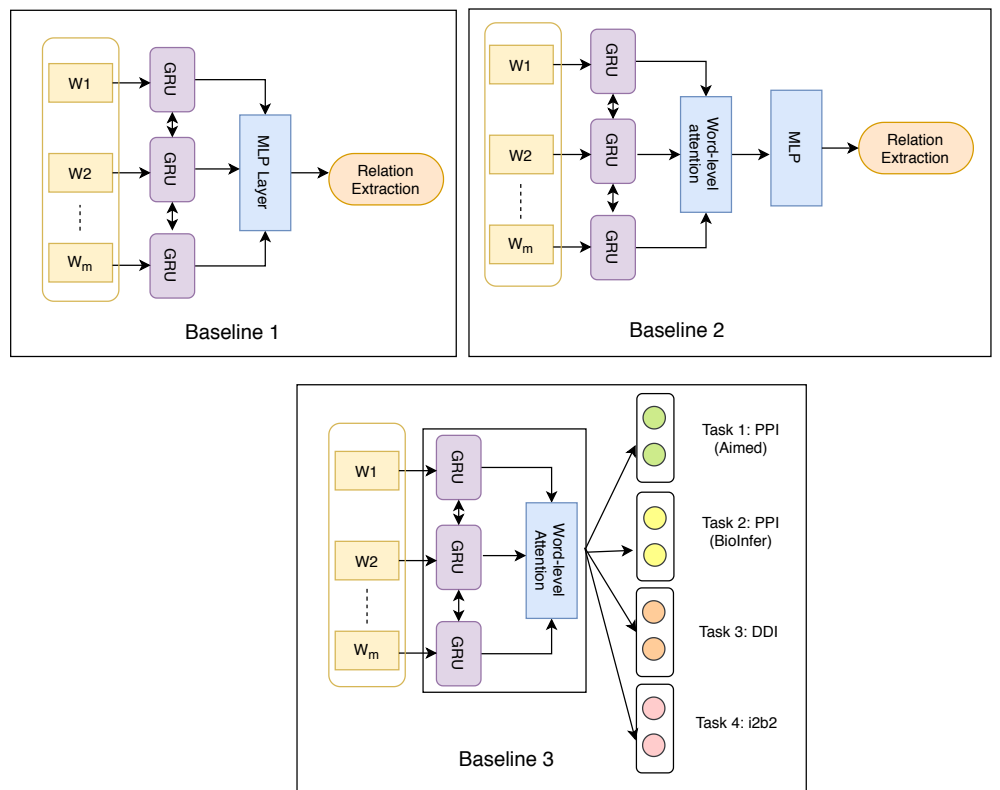

Fig. 2: Baseline models for various biomedical relation extraction tasks.

\begin{tabular}{|c|c|c|c|c|c|c|c|c|c|c|c|c|}
\hline Tasks & \multicolumn{3}{|c|}{ Baseline 1 } & \multicolumn{3}{|c|}{ Baseline 2} & \multicolumn{3}{|c|}{ Baseline 3} & \multicolumn{3}{|c|}{ Proposed Approach (MTL) } \\
\hline PPI-AiMED & 69.61 & 71.16 & 70.37 & 67.75 & 69.65 & 68.69 & 75.85 & 72.74 & 74.27 & 78.12 & 76.56 & 77.33 \\
\hline PPI-BioInfer & 71.29 & 70.95 & 71.12 & 68.47 & 69.03 & 68.75 & 75.44 & 73.79 & 74.61 & 76.69 & 75.98 & 76.33 \\
\hline DDI & 71.99 & 67.26 & 67.46 & 70.24 & 65.15 & 67.60 & 75.82 & 64.50 & 69.71 & 76.52 & 69.01 & 72.57 \\
\hline
\end{tabular}

TABLE 4: Evaluation results of proposed MTL model and baselines system. Performance is reported in terms of 'P': Precision, 'R': Recall, and 'F': macro-F1-Score. Baseline 1 is single task learning model based on Bi-GRU. Baseline 2 is STL model with Bi-GRU + word-level attention. Baseline 3 is MTL model with a shared Bi-GRU layer. All the results are statistically significant as $p$-value $<$ $0.05)$.

\begin{tabular}{cc}
\hline Hyper-parameters & Value \\
\hline Max sentence length & 60 \\
Embedding dimension & 200 \\
GRU Hidden State Dimension & 64 \\
Attention Size $\left(d_{z}\right)$ & 350 \\
Attention aspect Size $(a)$ & 5 \\
\# of hidden neuron in Feed-forward n/w & 100 \\
Activation & relu \\
Dropout rate & 0.3 \\
Output Activation & Soft-max \\
Optimizer & Adam Optimizer \\
Learning Rate & 0.001 \\
$\alpha$ & 1 \\
$\beta$ & 0.05 \\
\hline
\end{tabular}

TABLE 5: Optimal hyper-paramete values on proposed model

proposed approach. For DDI dataset, our proposed method attains improvements of 5.11 and 4.97 F1-Score points over Baseline 1 and Baseline 2, respectively. Lastly, for the i2b2-2010 clinical relation extraction dataset, the proposed method demonstrates the performance improvements of 1.56 and $1.06 \mathrm{~F} 1-$ Score points over Baseline 1 and Baseline 2, respectively.

We observe that our model outperforms Baseline 3 model, with 3.06, 1.72, 2.86, and 0.94 F-Score points on AiMED, BioInfer, DDI, and i2b2-2010 clinical relation extraction dataset respectively.

Overall, the findings achieved show that using self attention based adversarial multi-task learning to save the present knowledge in a shared layer is helpful for a new task.

Ablation Study: To analyze the impact of various component of our model, we perform the ablation study by removing one component from the proposed model and evaluate the performance on all the four tasks. We carried of two set of ablation study: (1) jointly training all the four dataset together (c.f. Table-6), and (2) training two similar task dataset i.e. AiMed and BioInfer together (since both share characteristics of having proteininteraction information ), DDI and i2b2-RE task together (c.f. Table-7). One possible reason for the i2b2-RE dataset to not be benefited from adversarial learning component is firstly because it is jointly trained with DDI corpus whose origins (clinical notes vs biomedical literature) and characteristics are not very similar to the i2b2-RE dataset in contrary to AiMED and Bionfer that are both protein-interacting datasets. Another reason is the huge difference between the samples in DDI (5550) and i2b2-RE (62254). In this case when the batches of the DDI dataset finish arriving, model will train only with the i2b2-RE dataset and could lead to the diminishing effect of the adversarial training.

Experimental results shows that removing self attention leads to the decrements in the performance of the model across all the task, in both the ablation study scenarios. However, we observe that adversarial learning was not that much effective in the ablation study 1 , where we jointly trained all the four dataset together. While for the ablation study 2, when we trained similar task 


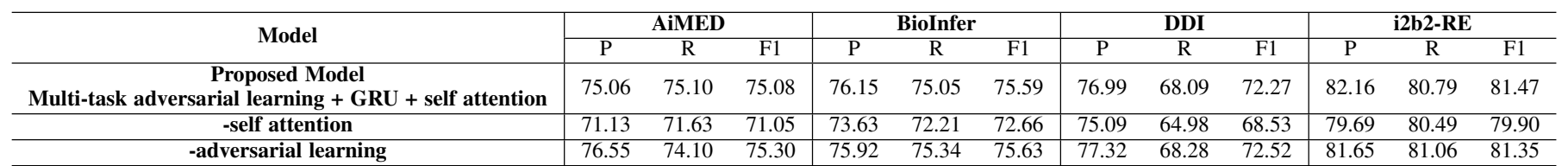

TABLE 6: Ablation study by jointly training all the four dataset together.

\begin{tabular}{|c|c|c|c|c|c|c|c|c|c|c|c|c|}
\hline \multirow{2}{*}{ Model } & \multicolumn{3}{|c|}{ AiMED } & \multicolumn{3}{|c|}{ BioInfer } & \multicolumn{3}{|c|}{ DDI } & \multicolumn{3}{|c|}{ i2b2-RE } \\
\hline & $\mathrm{P}$ & $\mathrm{R}$ & F1 & $\mathrm{P}$ & $\mathrm{R}$ & F1 & $\mathrm{P}$ & $\mathrm{R}$ & F1 & $\mathrm{P}$ & $\mathrm{R}$ & F1 \\
\hline $\begin{array}{c}\text { Proposed Model } \\
\text { Multi-task adversarial learning + GRU + self attention }\end{array}$ & 78.12 & 76.56 & 77.33 & 76.69 & 75.98 & 76.33 & 76.52 & 69.01 & 72.57 & 81.92 & 81.37 & 81.65 \\
\hline -self attention & 72.96 & 73.21 & 73.09 & 74.91 & 73.98 & 74.44 & 75.34 & 64.92 & 69.74 & 80.74 & 79.16 & 79.94 \\
\hline -adversarial learning & 78.26 & 75.43 & 76.82 & 76.57 & 75.72 & 76.14 & 77.92 & 67.31 & 72.23 & 83.68 & 81.02 & 82.33 \\
\hline
\end{tabular}

TABLE 7: Ablation study by training two similar dataset (AiMed + BioInfer) and (DDI+i2b2-RE) at a time.

\begin{tabular}{|c|c|c|c|c|c|c|c|}
\hline \multirow{2}{*}{ System } & \multirow{2}{*}{ Technique } & \multicolumn{3}{|c|}{ AiMED } & \multicolumn{3}{|c|}{ BioInfer } \\
\hline & & $\mathbf{P}$ & $\mathbf{R}$ & $\mathbf{F}$ & $\mathbf{P}$ & $\mathbf{R}$ & $\mathbf{F}$ \\
\hline Our System & MTL-Adversarial (Bi-GRU+ self-attention) & 78.12 & 76.56 & $\mathbf{7 7 . 3 3}$ & 76.69 & 75.98 & 76.33 \\
\hline$[\overline{40}]^{*}$ & LSTM $_{p r e}^{*}$ & 73.12 & 73.02 & 73.07 & 73.81 & 72.69 & 73.24 \\
\hline [41] & sdpCNN (SDP+CNN) & 64.80 & 67.80 & 66.00 & 73.40 & 77.00 & 75.20 \\
\hline$[56$ & Single kernel+ Multiple Parser+SVM & 59.10 & 57.60 & 58.10 & 63.61 & 61.24 & 62.40 \\
\hline [43] & McDepCNN & 67.3 & 60.1 & 63.5 & 62.7 & 68.2 & 65.3 \\
\hline [12] & Deep neural network & 51.5 & 63.4 & 56.1 & 53.9 & 72.9 & 61.6 \\
\hline
\end{tabular}

TABLE 8: Comparison with the SOTA techniques for PPI task on AiMed and BioInfer datasets. Performance is reported in terms of 'P': Precision, 'R': Recall, and 'F': F1-Score (macro). [40]* denotes the re-implementation of the systems proposed in [32] with the authors reported experimental setups using their publicly available source codes.

together, there was a drop in the performance across all the dataset. This shows that, adversarial learning is much helpful in the related task where the data distribution is similar.

\subsection{Comparative Analysis}

In this section, we will conduct the comparative analysis of our proposed method with the state-of-the-art (SOTA) model for all the three tasks.

- Protein-Protein Interaction: We used the SOTA methods for both datasets as presented in Table- 8 to compare our proposed model. The results show that the proposed model significantly outperforms the SOTA systems. From this we can conclude that our proposed multi-task model is more potent in extracting interacted protein pairs over the architecture based on CNN established in [41] and LSTM framework [40]. Our adversarial MTL model achieves a significant 4.26 F-score point increment over the LSTM based model [40] on AiMed dataset. In case of BioInfer dataset, our proposed model was able to achieve significant performance improvement of $3.09 \mathrm{~F}$-Score point over [40] as shown in Table-8 However, in comparison to [41], we could observe the improvement of $1.13 \mathrm{~F}$-Score points over the proposed model. This clearly demonstrates the effect of neural selfattention based adversarial learning in multi-task setting.

- Drug-Drug Interaction We compare our proposed model with SOTA DDI extraction techniques as shown in Table-9 Since, there was a difference in the dataset statistics, we reimplemented the system proposed by [87] using their publicly available source code on our DDI extraction dataset. Our multi-task adversarial Att-LSTM model obtains the significant performance improvement of 3.58 F-Score points over the state-of-the-art system [87] that exploited Bi-LSTM with attention mechanism. Also, the obtained experimental results illustrate that the model for the DDI task is benefited from other similar tasks, more specifically from the PPI tasks. It is because of the high semantic similarity between the sentences in DDI and PPI tasks.

- Medical Concept Relation We were unable to make direct comparison of our proposed approach with the systems participated in i2b2-2010 shared task due to the incomplete dataset. We compare our model with [78], as they also experimented with the same dataset. The results are reeported in Table 10. We obtain the performance improvements of 12.51, 9.91, 5.04, 11.39, and 5.65 F-Score points over [78] (irrespective of the use of additional linguistic features) for TeCP, TrCP, PIP, TrAP, and TeRP classes, respectively. This shows the usefulness of self attention based adversarial learning in multi-task setup which eventually gathers the complimentary features for medical concepts relations and other related tasks and improves the performance of the system.

\subsection{Error Analysis}

Here, we closely examine the various forms of errors with respect to the tasks that cause the mis-classification.

1) PPI: In case of the AiMED and BioInfer datasets, we observe that in a sentence with multiple protein mentions, our proposed model fails to identify properly the interacted pair. For example:

Sentence 1: "We screened proteins for interaction with PROTEIN and cloned the full-length cDNA of human PROTEIN which encoded 1225 amino acids." 


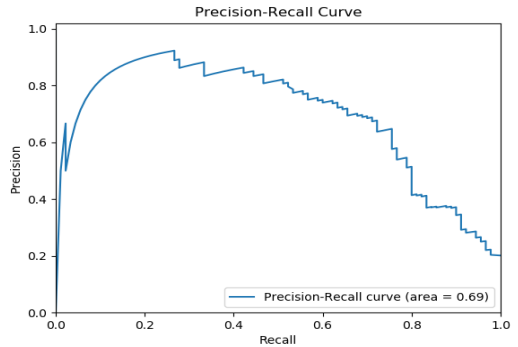

(a) PR curve for AiMed

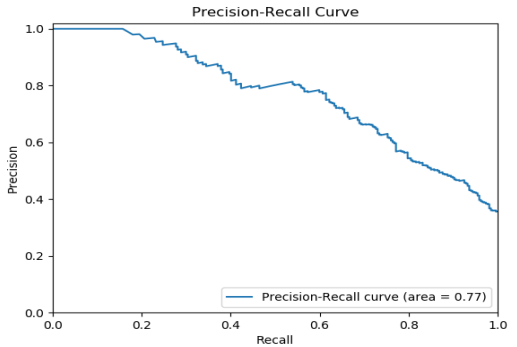

(b) PR curve for BioInfer

Fig. 3: The graphs (a) and (b) demonstrate the Precision-Recall curves for the binary classification dataset, AiMed and BioInfer.

\begin{abstract}
coassociation of cd26 protien with protien on the surface of human 1 lymphocytes thus the $\mathrm{c}$ terminal domain in protien appears to exert a negative control on binding of $\mathrm{p} 85$ thereby providing a possible regulatory mechanism characterization of the action of porcine protien on protien polymerization beta catenin or plakoglobin is associated directly with the cadherin protien binds to beta catenin plakoglobin

depression does nol always require druga therapy and drugb have no impacl on hot linked
if is better to avoid prescribing isoenzyme cyp dgddg inhibitors to women treated with tamoxifen for breast cancer especially druga such as drugb
btest scan revealed a metastatic lesion in the right of the but no bproblem abnormalities
\end{abstract}

Fig. 4: Heatmap of the attention weight distributions on the examples of different datasets. The intensity of the colour is increased with the increment in the attention weight.

Here, the actual label was true but our model predicted this as false. Repetitive mentions of proteins behave like a noise, than can inhibit the model to extract contextually relevant information. We also made an interesting observation that in the presence of the protein interacting words (such as 'bind', 'interact'), our model predicts the class label as 'interacting' (true). For example:

Sentence 1: "PROTEIN binding significantly increased hetero- and homo-oligomerization (except for the BR-II homo-oligomer, which binds ligand poorly in the absence of PROTEIN"

Sentence 2: "PROTEIN is a muscle-specific HLH protein that binds DNA in vitro as a heterodimer with several widely expressed HLH proteins, such as the PROTEIN gene products E12 and E47."

This is because these words often occur in the vicinity of the interacting protein mentions.

2) DDI: Apart from the highly imbalance dataset issue, our model fails to capture the exact relationship between the DDI pairs, where the lengths of the sentences were long. Another phenomenon that we observed as a source of mis-classification was that the "Int" type was often predicted as "Effect" class type. "Int" class describes the coarse classification, i.e., there exists interaction between two drugs. This implies that there could be a positive or negative outcome which forms the main cause of the system often getting confused between the "Int" and "Effect" class labels. For example:

Sentence 1: "Synergistic interaction between DRUGA and $D R U G B$ is sequence dependent in human non small lung cancer with EGFR TKIs resistant mutation."

We also found that some labels were incorrectly predicted because of class-specific keywords which exist in the sentence but are not related to the concerned entity pair. For example:

Sentence 1: "Interaction study of DRUGA and DRUGB with co administered drugs."

Sentence 2: "If in certain cases, an DRUGA is considered necessary, it may be advisable to replace tamoxifen with DRUGB."

In sentence 1 , the model got confused between the class label 'Int' and 'None', while in the sentence 2, our model incorrectly predicted the class label as 'Advice'.

3) Medical Concept Relation: We observe that due to close similarity between the class label ' $T e R P$ ' and ' $T e C P$ ', our model was found to be confused between these classes. For example:

Sentence 1: "TEST $x$-ray revealed no PROBLEM, no congestive heart failure."

In the given sentence, our model incorrectly predicted it as class ' $T e R P$ '.

We also observed that majority of the misclassification was between 'PIP' and 'NONE' class. For example:

Sentence 1: "There was PROBLEM atrophy and PROBLEM encephalomalacia." 


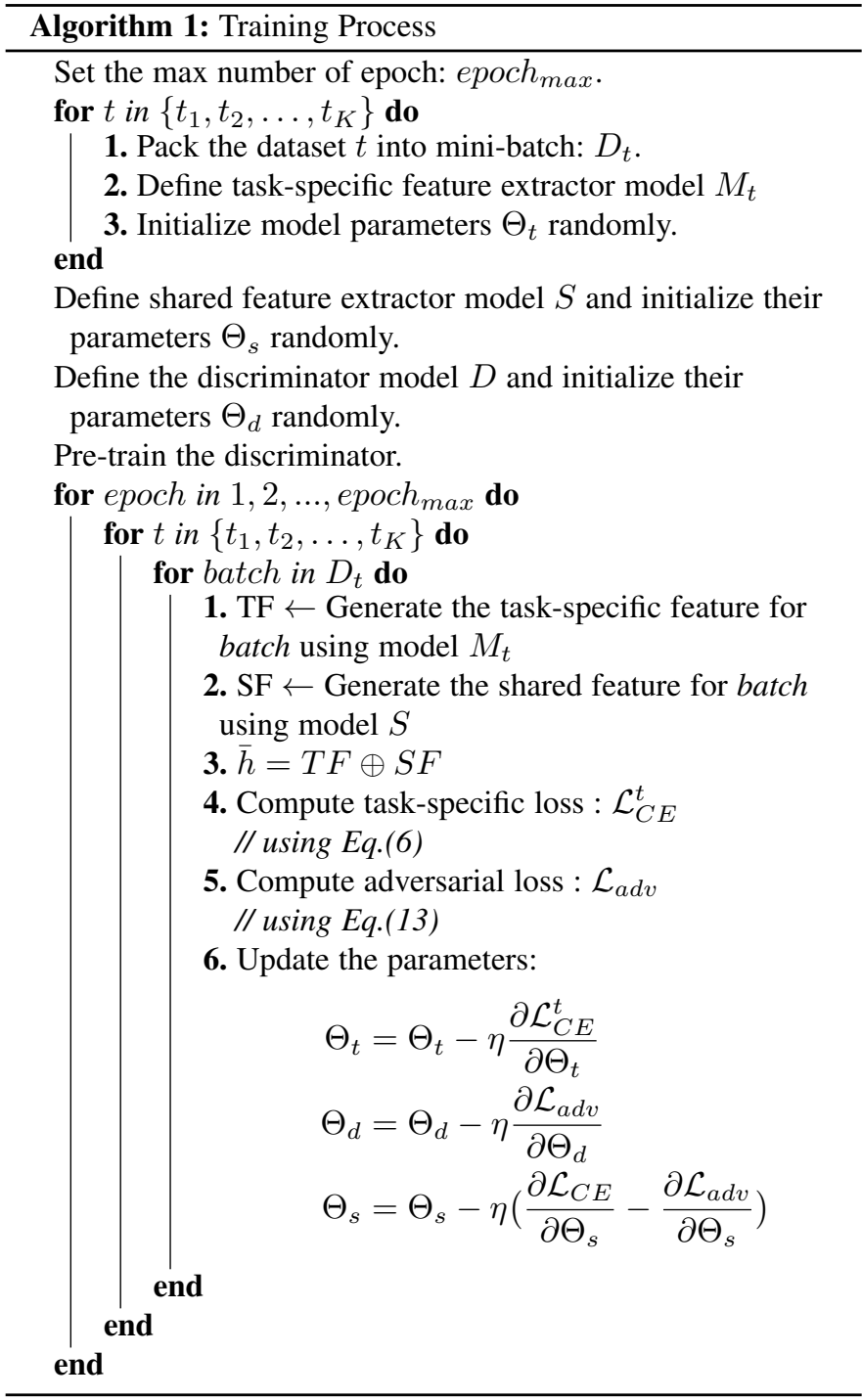

\subsection{Visual Analysis:}

We have carried out the visual analysis (in Figure 4) to get an intuitive understanding of attention weights in multi-task attention model. Each sentence in the figure shows the attention distribution in the form of heatmap for an instance of the corresponding dataset. The highlighted colours indicate the most relevant words in the sentence selected by the attention mechanism. For example in the sentence, "co-association of cd26 protein with the protein on the surface of human t lymphocytes", the model is able to provide more weights to "co-association of cd26 protein", which is relevant to correctly classify the given protein pair as interacted pair. For the binary classification datasets, AiMed and BioInfer, we also plot the Precision-Recall curve (c.f. Figure-3).

\section{Conclusion}

In this paper, we propose a unified multi-task learning approach that exploits the capabilities of adversarial learning approach for relation extraction from biomedical domain. We first experimented on three benchmark biomedical relation extraction tasks, i.e., protein-protein interaction, drug-drug interaction, and clinical relation extraction. For that, we utilized four popular datasets: AIMed, BioInfer, SemEval 2013 DDI shared task dataset and i2b2-2010 clinical relation dataset. We demonstrated that our model shows superior performance compared to state-of-the-art models for all the tasks.

Although our model has shown significant improvements over state-of-the-art methods on all the tasks, it was observed that our supervised model does not generalize well for the class label with the small instances. In future, we would like to develop a zeroshot learning method that could assist the model in the huge class imbalance issue.

Acknowledgement: Dr. Sriparna Saha gratefully acknowledges the Young Faculty Research Fellowship (YFRF) Award, supported by Visvesvaraya Ph.D. Scheme for Electronics and IT, Ministry of Electronics and Information Technology (MeitY), Government of India, being implemented by Digital India Corporation (formerly Media Lab Asia) for carrying out this research.

\section{REFERENCES}

[1] Z. Lu, "Pubmed and beyond: a survey of web tools for searching biomedical literature," Database, vol. 2011, 2011.

[2] R. Khare, R. Leaman, and Z. Lu, "Accessing biomedical literature in the current information landscape," Biomedical Literature Mining, pp. $11-31,2014$.

[3] S. Yadav, A. Ekbal, S. Saha, and P. Bhattacharyya, "Deep learning architecture for patient data de-identification in clinical records," in Proceedings of the clinical natural language processing workshop (ClinicalNLP), 2016, pp. 32-41.

[4] S. Yadav, A. Ekbal, and S. Saha, "Feature selection for entity extraction from multiple biomedical corpora: A pso-based approach," Soft Computing, vol. 22, no. 20, pp. 6881-6904, 2018.

[5] S. Yadav, A. Ekbal, S. Saha, and P. Bhattacharyya, "Entity extraction in biomedical corpora: An approach to evaluate word embedding features with pso based feature selection," in Proceedings of the 15th Conference of the European Chapter of the Association for Computational Linguistics: Volume 1, Long Papers, 2017, pp. 1159-1170.

[6] M. G. Kann, "Protein interactions and disease: computational approaches to uncover the etiology of diseases," Briefings in bioinformatics, vol. 8 , no. 5, pp. 333-346, 2007.

[7] R. C. Bunescu and R. J. Mooney, "A shortest path dependency kernel for relation extraction," in Proceedings of the conference on human language technology and empirical methods in natural language processing. Association for Computational Linguistics, 2005, pp. 724-731.

[8] A. Airola, S. Pyysalo, J. Björne, T. Pahikkala, F. Ginter, and T. Salakoski, "A graph kernel for protein-protein interaction extraction," in Proceedings of the workshop on current trends in biomedical natural language processing. Association for Computational Linguistics, 2008, pp. 1-9.

[9] P. Thomas, M. Neves, T. Rocktäschel, and U. Leser, "Wbi-ddi: drug-drug interaction extraction using majority voting," in Second Joint Conference on Lexical and Computational Semantics (* SEM), Volume 2: Proceedings of the Seventh International Workshop on Semantic Evaluation (SemEval 2013), vol. 2, 2013, pp. 628-635.

[10] Y. Liu, F. Wei, S. Li, H. Ji, M. Zhou, and H. Wang, "A dependencybased neural network for relation classification," arXiv preprint arXiv:1507.04646, 2015.

[11] S.-P. Choi, "Extraction of protein-protein interactions (ppis) from the literature by deep convolutional neural networks with various feature embeddings," Journal of Information Science, p. 0165551516673485 , 2016.

[12] Z. Zhao, Z. Yang, H. Lin, J. Wang, and S. Gao, "A protein-protein interaction extraction approach based on deep neural network," International Journal of Data Mining and Bioinformatics, vol. 15, no. 2, pp. 145-164, 2016.

[13] S. Yadav, A. Ekbal, S. Saha, P. Bhattacharyya, and A. Sheth, "Multitask learning framework for mining crowd intelligence towards clinical treatment," in Proceedings of the 2018 Conference of the North American Chapter of the Association for Computational Linguistics: Human Language Technologies, Volume 2 (Short Papers), vol. 2, 2018, pp. 271-277.

[14] S. Yadav, A. Ekbal, S. Saha, and P. Bhattacharyya, "A unified multitask adversarial learning framework for pharmacovigilance mining," in Proceedings of the 57th Annual Meeting of the Association for Computational Linguistics, 2019, pp. 5234-5245. 


\begin{tabular}{|c|c|c|c|c|}
\hline System & Technique & $\mathbf{P}$ & $\mathbf{R}$ & $\mathbf{F}$ \\
\hline Our System & Multi-task adversarial learning + GRU+attention & 76.52 & 69.01 & 72.57 \\
\hline Joint AB-LSTM* ${ }^{*}[\overline{87}]$ & Bi-directional LSTM +attention & 71.84 & 66.88 & 68.99 \\
\hline Yi et al. ${ }^{+}|\overline{88}|$ & RNN+dynamic WE+ multi-attention & 73.67 & 70.79 & 72.20 \\
\hline Joint AB-LSTM $^{+}$[87] & Bi-directional LSTM +attention & 73.41 & 69.66 & 71.48 \\
\hline $\mathrm{DCNN}^{+}|\overline{89}| \longrightarrow$ & Dependency based CNN & 77.21 & 64.35 & 70.19 \\
\hline Liu et al. $\overline{-190}]$ & CNN & 75.72 & 64.66 & 69.75 \\
\hline $\mathrm{SCNN}^{+}|64|$ & Two stage syntax CNN & 72.5 & 65.1 & 68.6 \\
\hline
\end{tabular}

TABLE 9: Comparison with the SOTA techniques for DDI task. Performance is reported in terms of 'P': Precision, 'R': Recall, and 'F': F1-Score (macro). * denotes the re-implementations of the systems proposed in [32] with the authors' reported experimental setups using their publicly available source code. ${ }^{+}$denotes that the reported results may not be directly comparable with our proposed system because of the difference in dataset statistics.

\begin{tabular}{l|l|l|l|l}
\hline System & Sahu et al. $[\overline{78}$ & \multicolumn{3}{|c}{ Proposed Approach } \\
\hline Relation-types & F & P & R & F \\
\hline TeCP & 50.56 & 62.89 & 63.43 & $\mathbf{6 3 . 0 7}$ \\
\hline TrCP & 56.44 & 68.44 & 64.52 & $\mathbf{6 6 . 3 5}$ \\
\hline PIP & 64.92 & 73.20 & 67.17 & $\mathbf{6 9 . 9 6}$ \\
\hline TrAP & 69.23 & 79.10 & 82.28 & $\mathbf{8 0 . 6 2}$ \\
\hline TeRP & 81.25 & 87.31 & 86.56 & $\mathbf{8 6 . 9 0}$ \\
\hline
\end{tabular}

actions on Multimedia Computing, Communications, and Applications (TOMM), vol. 16, no. 1s, pp. 1-21, 2020

[28] C. Blaschke, M. A. Andrade, C. A. Ouzounis, and A. Valencia, "Automatic extraction of biological information from scientific text: proteinprotein interactions." in Ismb, vol. 7, 1999, pp. 60-67.

[29] T. Ono, H. Hishigaki, A. Tanigami, and T. Takagi, "Automated extraction of information on protein-protein interactions from the biological literature," Bioinformatics, vol. 17, no. 2, pp. 155-161, 2001.

TABLE 10: Comparison with the SOTA techniques for i2b2-2010 clinical relation extraction task. For the fair comparison, we have reported the weighted F-Score.

[15] G. Crichton, S. Pyysalo, B. Chiu, and A. Korhonen, "A neural network multi-task learning approach to biomedical named entity recognition," BMC Bioinformatics, vol. 18, no. 1, p. 368, Aug 2017. [Online]. Available: https://doi.org/10.1186/s12859-017-1776-8

[16] I. Goodfellow, J. Pouget-Abadie, M. Mirza, B. Xu, D. Warde-Farley, S. Ozair, A. Courville, and Y. Bengio, "Generative adversarial nets," in Advances in neural information processing systems, 2014, pp. 2672 2680.

[17] K. Cho, B. Van Merriënboer, D. Bahdanau, and Y. Bengio, "On the properties of neural machine translation: Encoder-decoder approaches," arXiv preprint arXiv:1409.1259, 2014.

[18] S. Hochreiter and J. Schmidhuber, "Long short-term memory," Neural computation, vol. 9, no. 8, pp. 1735-1780, 1997.

[19] S. Pyysalo, A. Airola, J. Heimonen, J. Björne, F. Ginter, and T. Salakoski, "Comparative analysis of five protein-protein interaction corpora," in BMC bioinformatics, vol. 9, no. 3. BioMed Central, 2008, p. S6.

[20] I. Segura-Bedmar, P. Martínez, and M. H. Zazo, "Semeval-2013 task 9 : Extraction of drug-drug interactions from biomedical texts (ddiextraction 2013)," in Second Joint Conference on Lexical and Computational Semantics (* SEM), Volume 2: Proceedings of the Seventh International Workshop on Semantic Evaluation (SemEval 2013), vol. 2, 2013, pp. 341-350.

[21] Ö. Uzuner, B. R. South, S. Shen, and S. L. DuVall, "2010 i2b2/va challenge on concepts, assertions, and relations in clinical text," Journal of the American Medical Informatics Association, vol. 18, no. 5, pp. 552-556, 2011.

[22] M. E. Savery, W. J. Rogers, M. Pillai, J. G. Mork, and D. DemnerFushman, "Chemical entity recognition for medline indexing," AMIA Summits on Translational Science Proceedings, vol. 2020, p. 561, 2020.

[23] T. R. Goodwin and D. Demner-Fushman, "A customizable deep learning model for nosocomial risk prediction from critical care notes with indirect supervision," Journal of the American Medical Informatics Association, vol. 27, no. 4, pp. 567-576, 2020.

[24] A. Srivastava, A. Ekbal, S. Saha, P. Bhattacharyya et al., "A recurrent neural network architecture for de-identifying clinical records," in Proceedings of the 13th international conference on natural language processing, 2016, pp. 188-197.

[25] S. Yadav, A. Ekbal, and S. Saha, "Information theoretic-pso-based feature selection: an application in biomedical entity extraction," Knowledge and Information Systems, vol. 60, no. 3, pp. 1453-1478, 2019.

[26] A. Ekbal, S. Saha, P. Bhattacharyya et al., "A deep learning architecture for protein-protein interaction article identification," in 2016 23rd International Conference on Pattern Recognition (ICPR). IEEE, 2016, pp. 3128-3133.

[27] S. Yadav, P. Ramteke, A. Ekbal, S. Saha, and P. Bhattacharyya, "Exploring disorder-aware attention for clinical event extraction," ACM Trans-

[30] R. Bunescu, R. Ge, R. J. Kate, E. M. Marcotte, R. J. Mooney, A. K. Ramani, and Y. W. Wong, "Comparative experiments on learning information extractors for proteins and their interactions," Artificial intelligence in medicine, vol. 33, no. 2, pp. 139-155, 2005.

[31] Y. Miyao, K. Sagae, R. Sætre, T. Matsuzaki, and J. Tsujii, "Evaluating contributions of natural language parsers to protein-protein interaction extraction," Bioinformatics, vol. 25, no. 3, pp. 394-400, 2008

[32] N. Daraselia, A. Yuryev, S. Egorov, S. Novichkova, A. Nikitin, and I. Mazo, "Extracting human protein interactions from medline using a full-sentence parser," Bioinformatics, vol. 20, no. 5, pp. 604-611, 2004.

[33] G. Erkan, A. Özgür, and D. R. Radev, "Semi-supervised classification for extracting protein interaction sentences using dependency parsing." in EMNLP-CoNLL, vol. 7, 2007, pp. 228-237.

[34] R. Sætre, K. Sagae, and J. Tsujii, "Syntactic features for protein-protein interaction extraction.” LBM (Short Papers), vol. 319, 2007.

[35] A. Airola, S. Pyysalo, J. Björne, T. Pahikkala, F. Ginter, and T. Salakoski, "All-paths graph kernel for protein-protein interaction extraction with evaluation of cross-corpus learning," BMC bioinformatics, vol. 9, no. 11, p. S2, 2008.

[36] M. Zhang, J. Zhang, J. Su, and G. Zhou, "A composite kernel to extract relations between entities with both flat and structured features," in Proceedings of the 21st International Conference on Computational Linguistics and the 44th annual meeting of the Association for Computational Linguistics. Association for Computational Linguistics, 2006, pp. $825-832$.

[37] S. Kim, J. Yoon, J. Yang, and S. Park, "Walk-weighted subsequence kernels for protein-protein interaction extraction," BMC bioinformatics, vol. 11, no. 1, p. 107, 2010.

[38] Y.-C. Chang, C.-H. Chu, Y.-C. Su, C. C. Chen, and W.-L. Hsu, "Pipe: a protein-protein interaction passage extraction module for biocreative challenge," Database, vol. 2016, 2016.

[39] S. Yadav, A. Kumar, A. Ekbal, S. Saha, and P. Bhattacharyya, "Feature assisted bi-directional lstm model for protein-protein interaction identification from biomedical texts," arXiv preprint arXiv:1807.02162, 2018.

[40] Y.-L. Hsieh, Y.-C. Chang, N.-W. Chang, and W.-L. Hsu, "Identifying protein-protein interactions in biomedical literature using recurrent neural networks with long short-term memory," in Proceedings of the eighth international joint conference on natural language processing (volume 2: short papers), 2017, pp. 240-245.

[41] L. Hua and C. Quan, "A shortest dependency path based convolutional neural network for protein-protein relation extraction," BioMed Research International, vol. 2016, 2016.

[42] S.-P. Choi, "Extraction of protein-protein interactions (ppis) from the literature by deep convolutional neural networks with various feature embeddings," Journal of Information Science, vol. 44, no. 1, pp. 60-73, 2018.

[43] Y. Peng and Z. Lu, "Deep learning for extracting protein-protein interactions from biomedical literature," arXiv preprint arXiv:1706.01556, 2017.

[44] S. Yadav, A. Ekbal, S. Saha, A. Kumar, and P. Bhattacharyya, "Feature assisted stacked attentive shortest dependency path based bi-lstm model for protein-protein interaction," Knowledge-Based Systems, vol. 166, pp. 18-29, 2019. 
[45] M. Ahmed, J. Islam, M. R. Samee, and R. E. Mercer, "Identifying protein-protein interaction using tree 1 stm and structured attention," in 2019 IEEE 13th International Conference on Semantic Computing (ICSC). IEEE, 2019, pp. 224-231.

[46] S. K. Sahu and A. Anand, "Drug-drug interaction extraction from biomedical texts using long short-term memory network," Journal of Biomedical Informatics, vol. 86, pp. 15 - 24, 2018. [Online]. Available: http://www.sciencedirect.com/science/article/pii/S1532046418301606

[47] T. Bobic, J. Fluck, and M. Hofmann-Apitius, "Scai: Extracting drug-drug interactions using a rich feature vector," in Second Joint Conference on Lexical and Computational Semantics (* SEM), Volume 2: Proceedings of the Seventh International Workshop on Semantic Evaluation (SemEval 2013), 2013, pp. 675-683.

[48] N. Hailu, L. E. Hunter, and K. B. Cohen, "Ucolorado_som: extraction of drug-drug interactions from biomedical text using knowledge-rich and knowledge-poor features," in Second Joint Conference on Lexical and Computational Semantics (*SEM), Volume 2: Proceedings of the Seventh International Workshop on Semantic Evaluation (SemEval 2013), 2013, pp. 684-688.

[49] J. Björne, S. Kaewphan, and T. Salakoski, "Uturku: drug named entity recognition and drug-drug interaction extraction using svm classification and domain knowledge," in Second Joint Conference on Lexical and Computational Semantics (*SEM), Volume 2: Proceedings of the Seventh International Workshop on Semantic Evaluation (SemEval 2013), 2013, pp. 651-659.

[50] M. Rastegar-Mojarad, R. D. Boyce, and R. Prasad, "Uwm-triads: classifying drug-drug interactions with two-stage svm and post-processing," in Second Joint Conference on Lexical and Computational Semantics (* SEM), Volume 2: Proceedings of the Seventh International Workshop on Semantic Evaluation (SemEval 2013), 2013, pp. 667-674.

[51] S. Kim, H. Liu, L. Yeganova, and W. J. Wilbur, "Extracting drugdrug interactions from literature using a rich feature-based linear kernel approach," Journal of biomedical informatics, vol. 55, pp. 23-30, 2015.

[52] B. Bokharaeian and A. Díaz, "Nil_ucm: Extracting drug-drug interactions from text through combination of sequence and tree kernels," in Second Joint Conference on Lexical and Computational Semantics (* SEM), Volume 2: Proceedings of the Seventh International Workshop on Semantic Evaluation (SemEval 2013), 2013, pp. 644-650.

[53] M. F. M. Chowdhury and A. Lavelli, "Fbk-irst: A multi-phase kernel based approach for drug-drug interaction detection and classification that exploits linguistic information," in Second Joint Conference on Lexical and Computational Semantics (* SEM), Volume 2: Proceedings of the Seventh International Workshop on Semantic Evaluation (SemEval 2013), vol. 2, 2013, pp. 351-355.

[54] R. Harpaz, A. Callahan, S. Tamang, Y. Low, D. Odgers, S. Finlayson, K. Jung, P. LePendu, and N. H. Shah, "Text mining for adverse drug events: the promise, challenges, and state of the art," Drug safety, vol. 37, no. 10, pp. 777-790, 2014.

[55] R. Xu and Q. Wang, "Large-scale automatic extraction of side effects associated with targeted anticancer drugs from full-text oncological articles," Journal of biomedical informatics, vol. 55, pp. 64-72, 2015.

[56] L. Qian and G. Zhou, "Tree kernel-based protein-protein interaction extraction from biomedical literature," Journal of biomedical informatics, vol. 45, no. 3, pp. 535-543, 2012.

[57] À. Bravo, J. Piñero, N. Queralt-Rosinach, M. Rautschka, and L. I. Furlong, "Extraction of relations between genes and diseases from text and large-scale data analysis: implications for translational research," BMC bioinformatics, vol. 16, no. 1, p. 55, 2015.

[58] B. Rink, S. Harabagiu, and K. Roberts, "Automatic extraction of relations between medical concepts in clinical texts," Journal of the American Medical Informatics Association, vol. 18, no. 5, pp. 594-600, 2011.

[59] A. Airola, S. Pyysalo, J. Björne, T. Pahikkala, F. Ginter, and T. Salakoski, "All-paths graph kernel for protein-protein interaction extraction with evaluation of cross-corpus learning," $B M C$ Bioinformatics, vol. 9, no. 11, p. S2, Nov 2008. [Online]. Available: https://doi.org/10.1186/1471-2105-9-S11-S2

[60] D. Tikk, P. Thomas, P. Palaga, J. Hakenberg, and U. Leser, "A comprehensive benchmark of kernel methods to extract protein-protein interactions from literature," PLoS computational biology, vol. 6, no. 7, p. e1000837, 2010.

[61] C. Giuliano, A. Lavelli, and L. Romano, "Exploiting shallow linguistic information for relation extraction from biomedical literature." in $E A C L$, vol. 18, no. 2006. Citeseer, 2006, pp. 401-408.

[62] P. Thomas, M. Neves, I. Solt, D. Tikk, and U. Leser, "Relation extraction for drug-drug interactions using ensemble learning," Training, vol. 4, no. 2,402, pp. 21-425, 2011.
[63] M. F. M. Chowdhury, A. B. Abacha, A. Lavelli, and P. Zweigenbaum, "Two different machine learning techniques for drug-drug interaction extraction," Challenge task on drug-drug interaction extraction, pp. 1926, 2011.

[64] Z. Zhao, Z. Yang, L. Luo, H. Lin, and J. Wang, "Drug drug interaction extraction from biomedical literature using syntax convolutional neural network," Bioinformatics, vol. 32, no. 22, pp. 3444-3453, 2016.

[65] W. Zheng, H. Lin, L. Luo, Z. Zhao, Z. Li, Y. Zhang, Z. Yang, and J. Wang, "An attention-based effective neural model for drug-drug interactions extraction," BMC bioinformatics, vol. 18, no. 1, p. 445, 2017.

[66] R. Kavuluru, A. Rios, and T. Tran, "Extracting drug-drug interactions with word and character-level recurrent neural networks," in 2017 IEEE International Conference on Healthcare Informatics (ICHI). IEEE, 2017, pp. 5-12.

[67] W. Wang, X. Yang, C. Yang, X. Guo, X. Zhang, and C. Wu, "Dependency-based long short term memory network for drug-drug interaction extraction," BMC bioinformatics, vol. 18, no. 16, p. 578, 2017.

[68] M. Asada, M. Miwa, and Y. Sasaki, "Extracting drug-drug interactions with attention cnns," in BioNLP 2017, 2017, pp. 9-18.

[69] S. Lim, K. Lee, and J. Kang, "Drug drug interaction extraction from the literature using a recursive neural network," PloS one, vol. 13, no. 1, p. e0190926, 2018.

[70] B. de Bruijn, C. Cherry, S. Kiritchenko, J. Martin, and X. Zhu, "Nrc at i2b2: one challenge, three practical tasks, nine statistical systems, hundreds of clinical records, millions of useful features," in Proceedings of the 2010 i2b2/VA Workshop on Challenges in Natural Language Processing for Clinical Data. Boston, MA, USA: i2b2, 2010.

[71] N. Kang, R. J. Barendse, Z. Afzal, B. Singh, M. J. Schuemie, E. M. van Mulligen, and J. A. Kors, "Erasmus mc approaches to the $\mathrm{i} 2 \mathrm{~b} 2$ challenge," in Proceedings of the 2010 i2b2/VA workshop on challenges in natural language processing for clinical data. i2b2, Boston, MA, USA, 2010.

[72] M. Jiang, Y. Chen, M. Liu, T. Rosenbloom, S. Mani, J. C. Denny, and $\mathrm{H}$. Xu, "Hybrid approaches to concept extraction and assertion classification-vanderbilt's systems for 2010 i2b2 nlp challenge," in Proceedings of the 2010 i2b2/VA Workshop on Challenges in Natural Language Processing for Clinical Data. Boston, MA, USA: i2b2, 2010.

[73] C. Grouin, A. Ben Abacha, D. Bernhard, B. Cartoni, L. Deleger, B. Grau, A.-L. Ligozat, A.-L. Minard, S. Rosset, and P. Zweigenbaum, "CARAMBA: Concept, Assertion, and Relation Annotation using Machine-learning Based Approaches," in i2b2 Medication Extraction Challenge Workshop, Washington, United States, Nov. 2010, pp. -. [Online]. Available: https://hal.archives-ouvertes.fr/hal-00795663

[74] K. Roberts, B. Rink, and S. Harabagiu, "Extraction of medical concepts, assertions, and relations from discharge summaries for the fourth i2b2/va shared task," in Proceedings of the 2010 i2b2/VA Workshop on Challenges in Natural Language Processing for Clinical Data. Boston, MA, USA: $i 2 b 2,2010$.

[75] S. Jonnalagadda, T. Cohen, S. Wu, and G. Gonzalez, "Enhancing clinical concept extraction with distributional semantics," Journal of biomedical informatics, vol. 45, no. 1, pp. 129-140, 2012.

[76] B. De Bruijn, C. Cherry, S. Kiritchenko, J. Martin, and X. Zhu, "Machine-learned solutions for three stages of clinical information extraction: the state of the art at $\mathrm{i} 2 \mathrm{~b} 2$ 2010," Journal of the American Medical Informatics Association, vol. 18, no. 5, pp. 557-562, 2011.

[77] D. Demner-Fushman, E. Apostolova, R. Islamaj Dogan, F.-M. Lang, J. Mork, A. Neveol, S. Shooshan, M. Simpson, and A. Aronson, "Nlm's system description for the fourth i2b2/va challenge," in Proceedings of the 2010 i2b2/VA Workshop on Challenges in Natural Language Processing for Clinical Data. Boston, MA, USA: i2b2, 2010.

[78] S. K. Sahu, A. Anand, K. Oruganty, and M. Gattu, "Relation extraction from clinical texts using domain invariant convolutional neural network," arXiv preprint arXiv: 1606.09370, 2016.

[79] Y. Luo, "Recurrent neural networks for classifying relations in clinical notes," Journal of Biomedical Informatics, vol. 72, pp. 85 - 95, 2017. [Online]. Available: http://www.sciencedirect.com/science/article/ pii/S1532046417301624

[80] B. Rosario and M. A. Hearst, "Classifying semantic relations in bioscience texts," in Proceedings of the 42nd annual meeting on association for computational linguistics. Association for Computational Linguistics, 2004, p. 430.

[81] M. Bundschus, M. Dejori, M. Stetter, V. Tresp, and H.-P. Kriegel, "Extraction of semantic biomedical relations from text using conditional random fields," BMC bioinformatics, vol. 9, no. 1, p. 207, 2008.

[82] D. Ningthoujam, S. Yadav, P. Bhattacharyya, and A. Ekbal, "Relation extraction between the clinical entities based on the shortest dependency path based lstm," arXiv preprint arXiv:1903.09941, 2019. 
[83] Z. Lin, M. Feng, C. N. d. Santos, M. Yu, B. Xiang, B. Zhou, and Y. Bengio, "A structured self-attentive sentence embedding," arXiv preprint arXiv:1703.03130, 2017.

[84] P. Liu, X. Qiu, and X. Huang, "Adversarial multi-task learning for text classification," in Proceedings of the 55th Annual Meeting of the Association for Computational Linguistics (Volume 1: Long Papers). Vancouver, Canada: Association for Computational Linguistics, July 2017, pp. 1-10. [Online]. Available: http://aclweb.org/anthology/ P17-1001

[85] Y. Ganin and V. Lempitsky, "Unsupervised domain adaptation by backpropagation," arXiv preprint arXiv:1409.7495, 2014.

[86] Z. Yang, D. Yang, C. Dyer, X. He, A. Smola, and E. Hovy, "Hierarchical attention networks for document classification," in Proceedings of the 2016 Conference of the North American Chapter of the Association for Computational Linguistics: Human Language Technologies, 2016, pp. $1480-1489$.

[87] S. K. Sahu and A. Anand, "Drug-drug interaction extraction from biomedical text using long short term memory network," arXiv preprint arXiv:1701.08303, 2017.

[88] Z. Yi, S. Li, J. Yu, Y. Tan, Q. Wu, H. Yuan, and T. Wang, "Drugdrug interaction extraction via recurrent neural network with multiple attention layers," in International Conference on Advanced Data Mining and Applications. Springer, 2017, pp. 554-566.

[89] S. Liu, K. Chen, Q. Chen, and B. Tang, "Dependency-based convolutional neural network for drug-drug interaction extraction," in Bioinformatics and Biomedicine (BIBM), 2016 IEEE International Conference on. IEEE, 2016, pp. 1074-1080.

[90] S. Liu, B. Tang, Q. Chen, and X. Wang, "Drug-drug interaction extraction via convolutional neural networks," Computational and mathematical methods in medicine, vol. 2016, 2016. 